\section{Transplantation \\ PTU-051 DONOR MORBIDITY FOLLOWING LIVING DONOR LIVER TRANSPLANTATION (LDLT): OUTCOME FROM A SMALL VOLUME CENTRE}

doi:10.1136/gutjnl-2012-302514c.51

A Hakeem, ${ }^{*}$ S Raza, M Reddy, J Jeffery, E Hidalgo, M Attia, S Pollard, G Toogood, C Millson, J P A Lodge, K R Prasad. Department of HPB and Liver Transplantation, St James's University Hospital NHS Trust, Leeds, UK

Introduction Living Donor Liver Transplantation (LDLT) has grown immensely in certain countries over the last few years, whereas in West the growth remains static and low volume. Donor morbidity following LDLT has been reported in the median range of 15\%-30\% in various studies. One of the limiting factor for the growth of LDLT has been the concerns with morbidity and mortality during a centres initial experience. We report the donor outcome from a small volume centre, so as to assess if there is an impact on volume with donor outcome.

Methods Between June 2007 and December 2011, 25 LDLT procedures were carried out in our unit. The criteria for donor selection included age, fitness for surgery and remnant liver volume. Donor demographics, graft type, complications, length of stay and overall survival were extracted. Donor morbidity was assessed objectively using the modified Clavien-Dindo classification. Continuous variables are expressed as mean $\pm \mathrm{SD}$.

Results The mean donor age was $38.4 \pm 11.0$ years and donor BMI was $23.9 \pm 2.8 \mathrm{~kg} / \mathrm{cm}^{2}$. Two donors were abandoned on table due to complex arterial and biliary anatomy respectively. The graft type was right lobe $(n=12,52.2 \%)$, left lateral lobe $(n=10,43.5 \%)$ and left lobe $(n=1,4.3 \%)$. The graft weight was $528.5 \pm 258.9 \mathrm{~g}$. The morbidity was $21.7 \%(n=5)$. There were three Clavien grade II complications (wound infection, urinary infection and unknown sepsis needing antibiotics). One patient needed laparotomy for haemorrhage (Grade IIIb) and another patient had ultrasound guided drainage of subphrenic collection (Grade IIIa). None of our patients had any post-operative blood transfusion. The length of hospital stay was $7.9 \pm 2.8$ days. At the end of median follow-up of 21.4 months, all our donors were alive, with no long-term morbidity.

Conclusion Our experience shows that donor hepatectomy for Living Donor Liver Transplantation is a safe procedure in a small volume unit. Our donor morbidity of $21.7 \%$ is comparable or better than most high volume centres across the world. Number of procedures performed by the unit shouldn't be an hindrance to the introduction of live donor liver programme.

Competing interests None declared.

\section{PTU-052 IMPACT OF BODY MASS INDEX ON THE OUTCOME OF LIVER TRANSPLANTATION IN CHILDREN}

doi:10.1136/gutjnl-2012-302514c.52

${ }^{1} \mathrm{~A}$ Hakeem, ${ }^{*}{ }^{1} \mathrm{G}$ Marangoni, ${ }^{1} \mathrm{~S}$ Chapman, ${ }^{1} \mathrm{R}$ Young, ${ }^{1} \mathrm{~A}$ Nair, ${ }^{1} \mathrm{M}$ Attia, ${ }^{2} \mathrm{P}$ McClean, ${ }^{1} \mathrm{~K}$ R Prasad. ${ }^{1}$ Department of HPB and Liver Transplantation, St James's University Hospital NHS Trust, UK; ${ }^{2}$ Department of Paediatric Liver Transplantation, Leeds General Infirmary, Leeds, UK

Introduction The impact of poor nutritional status on the outcome following liver transplantation in children is well recognised. Recently studies in adults and children have reported variable effects of obesity on post transplant morbidity and mortality. This study examines the impact of pretransplant body mass index (BMI) on the outcome of liver transplantation in the current era in consecutive children from a single centre.
Methods Data were retrieved from a prospectively maintained institutional database from 1994 to 2009. Patients were stratified into five BMI categories established by the WHO according to their Z scores: severe thinness $(-3)$, thinness $(-2)$, normal weight $(0)$, overweight $(+2)$ and obese $(+3)$. Primary outcome was to evaluate post-operative morbidity and secondary measures were overall patient and graft survival. Categorical variables were analysed by $\chi^{2}$, and continuous variables by one-way ANOVA. Kaplan-Meier curves were used to study patient and graft survival.

Results 146 paediatric liver transplants recipients were identified, of which 132 patients with height and weight data were included $12.2 \%$ of patients were underweight ( $Z$ score -2 and -3 ), $8.3 \%$ were overweight ( $Z$ score +2 and +3 ) and the remaining $79.5 \%$ were normal weight for age. The overall morbidity was higher in underweight recipients in comparison to normal weight $(81.2 \%$ vs $42.8 \%$, $\mathrm{p}=0.006$ ). Post-operative septic events were common in underweight recipients in comparison to normal weight recipients $(75.0 \%$ vs $25.7 \%, \mathrm{p}<0.001)$. Underweight patients had significantly longer intensive care stay than normal weight patients (mean 5.6 vs 3.1 days, $p=0.029$ ). The length of ventilation period was longer in underweight recipients in comparison to normal weight recipients (mean 3.4 vs 0.9 days, $\mathrm{p}=0.001$ ). There was no difference in the overall length of post-operative hospital stay between underweight and normal weight recipients (mean 34.2 vs 30.9 days, $p=0.602$ ) There was no difference in the post-operative septic events, ITU stay, ventilatory period and hospital stay between overweight and normal weight. There was no difference in overall graft $(p=0.949)$ and patient survival $(p=0.984)$ between the three groups

Conclusion This is the only reported UK series on BMI and outcome following paediatric liver transplantation. Despite current standards of peritransplant management we have demonstrated increased overall morbidity in underweight patients, with increased rates of post-operative septic complications, longer ventilatory period, and increased length of intensive care stay. However, unlike earlier studies, patient and graft survival were not affected. This study does not demonstrate any effect of obesity on post transplant morbidity or mortality.

Competing interests None declared.

\section{PTU-053 THE EFFECT OF HYPOXIC STRESS ON ACTIVATED HEPATIC STELLATE CELL CHEMOTAXIS IN-VITRO}

doi:10.1136/gutjnl-2012-302514c.53

${ }^{1} \mathrm{~A}$ Amer, ${ }^{* 2} \mathrm{D}$ Manas, ${ }^{1} \mathrm{M}$ Wright, ${ }^{2} \mathrm{~S}$ White. ${ }^{1}$ Institute of Cellular Medicine, Newcastle University, Newcastle upon Tyne, UK; ${ }^{2}$ Department of Hepato-Pancreatico-Biliary Surgery, Freeman Hospital, Newcastle upon Tyne, UK

Introduction Hepatic stellate cells (HSC) are believed to be the most important precursor of matrix-producing myofibroblasts that promote fibrosis following liver injury. In their activated form, HSCs migrate towards inflammatory foci in response to chemokines. When exposed to certain stresses, liver cells can express chemokines that control HSC migration. The aim of this study was to investigate the effect of hypoxia on chemokine expression by non-parenchymal liver cells using an in vitro model of ischaemia.

Methods Human HSCs, biliary epithelial cells (BECs) and Kupffer cells $(\mathrm{KC})$ were isolated from normal-appearing liver tissue obtained from liver resection specimens $(n=3)$. HSCs were cultured on either gel matrix or uncoated plastic in order to investigate both quiescent (qHSC) and activated (aHSC) phenotypes. Conditioned medium from each cell type was collected at various time points in culture after exposure to either normoxia $(21 \%$ O2) or hypoxia $(1 \%$ O2). For migration assays, fully activated human HSCs were used and were seeded on cell culture inserts exposed to conditioned medium from each cell type added to the lower compartment. Cells were then 
incubated for $20 \mathrm{~h}$ following which the migrated cells were fixed, stained and counted.

Results HSCs demonstrated greater migration towards medium obtained from HSCs cultured for 3 days under hypoxic conditions compared to normoxic HSCs $(p=0.06)$. This effect decreased with longer culture times, reaching levels significantly lower than baseline by day 7 for both groups $(p<0.0001)$. In contrast, medium obtained from HSCs cultured on gel matrix under normoxic conditions stimulated significantly higher HSC migration compared to hypoxic qHSCs, with a peak effect by day $5(\mathrm{p}<0.0001)$. Similarly, normoxic KCs stimulated significantly enhanced HSCs migration compared to hypoxic KCs $(p<0.0001)$. On the other hand, hypoxic BECs attracted significantly more HSCs on day 1 compared to normoxia, an effect that continued to rise on day $7(p=0.002)$.

Conclusion This study demonstrates that hypoxia in an in vitro model stimulates contrasting responses depending on cell type and activation state. KCs and qHSCs in a relatively high $\mathrm{O} 2$ state similar to that of reperfusion during liver surgery can promote chemotaxis of aHSCs possibly through the formation of reactive oxygen species. BECs and aHSCs appear to produce factors that negatively affect HSC chemotaxis as evident by below-baseline migration responses. Hypoxia attenuates this negative effect in vitro.

Competing interests None declared.

\section{PTU-054 TRANSJUGULAR INTRAHEPATIC PORTOSYSTEMIC SHUNTS FOLLOWING LIVER TRANSPLANTATION CAN BE ASSOCIATED WITH A GOOD PROGNOSIS: A SINGLE CENTRE EXPERIENCE}

doi:10.1136/gutjnl-2012-302514c.54

${ }^{1} B$ Hogan, * ${ }^{1} \mathrm{D}$ Thorburn, 'D Sharma, ${ }^{1} \mathrm{~B}$ Davidson, ${ }^{1} \mathrm{~J}$ P O'Beirne, 'A K Burroughs, ${ }^{2} \mathrm{D}$ Yu, ${ }^{1} \mathrm{D}$ W Patch. ${ }^{1}$ Sheila Sherlock Liver Centre, Royal Free Hospital, London, UK; ${ }^{2}$ Department of Interventional Radiology, Royal Free Hospital, London, UK

Introduction Transjugular intrahepatic portosystemic shunt (TIPS) is indicated in the management of portal vein thrombosis or stenosis, portal hypertension and for veno-occlusive disease in post-liver transplant (LT) patients. Previous series have reported 1-year mortality rates of $14 \%-67 \%$. A MELD score $>15$ at the time of insertion may indicate a poor long term prognosis. We aimed to evaluate the safety of TIPS after LT at a UK tertiary referral centre.

Methods We retrospectively analysed data from the Royal Free Hospital TIPS database between 1st January 1991 and the 31st January 2011. All patients who had undergone TIPS following LT were included.

Results During the period studied 629 patients received a TIPS. In the same period 1192 liver transplant operations were performed. 10 TIPS were inserted into patients following LT for recurrent cirrhosis with refractory ascites (4), veno-occlusive disease (3) and portal vein thrombosis (3). The original indications for transplantation were PSC (3), PBC (3), Hepatitis C (1), Autoimmune (1), Primary Oxalosis (1) and Acute Liver Failure (1). We noted a median survival of 38 months. Survival at 1 and 5 years was $100 \%$ and $60 \%$ respectively. The median MELD at the time of TIPS insertion was 12 (range 7-19). No correlation between the MELD score at the time of TIPS insertion and survival was demonstrated $(p=0.62)$.

Conclusion These results suggest that TIPS can be performed safely after LT and that survival rates better than those previously reported can be achieved. We suggest TIPS should be used in carefully selected candidates following LT as a definitive treatment for patients not suitable for re-transplant or as a bridge to re-transplantation. The alternative of re-transplantation should always be considered prior to TIPS insertion where indicated.

\section{Competing interests None declared.}

\section{REFERENCES}

1. Saad WEA, et al. Transjugular intrahepatic portosystemic shunts in liver transplant recipients for management of refractory ascites: clinical outcome. J Vasc Interv Radiol 2010;21:218-23.

2. Kim JJ, et al. Transjugular intrahepatic portosystemic shunts in liver transplant recipients. Liver Int 2008;28:240-8.

3. Feyssa $\mathbf{E}$, et al. MELD score less than 15 predicts prolonged survival after transjugular intrahepatic portosystemic shunt for refractory ascites after liver transplantation. Transplantation 2011;91:786-92.

\section{PTU-055 THE ASSOCIATION OF CO-EXISTENT NON-ISCHAEMIC CARDIAC DISEASE WITH MORBIDITY AND MORTALITY FOLLOWING LIVER TRANSPLANTATION}

doi:10.1136/gutjnl-2012-302514c.55

${ }^{1} \mathrm{H}$ Steed, ${ }^{*}{ }^{1} \mathrm{~J}$ A Leithead, ${ }^{1} \mathrm{~K}$ Kandiah, ${ }^{2} \mathrm{~B}$ K Gunson, ${ }^{1} \mathrm{~J}$ W Ferguson. ${ }^{1}$ Liver Unit, Queen Elizabeth Hospital, Birmingham, UK; ${ }^{2}$ NIHR Biomedical Research Unit and Center for Research, University of Birmingham, Birmingham, UK

Introduction The risk stratification of potential liver transplant candidates with co-existent non-ischaemic cardiac disease (CD) is particularly challenging given the masking effect of the haemodynamic dysfunction of advanced portal hypertension. Whether such patients have altered outcomes following liver transplantation remains unknown. Our aim was, therefore, to examine the effects of mild and moderate non-ischaemic $\mathrm{CD}$ on post liver transplant morbidity and mortality.

Methods Single-centre study of patients undergoing first liver transplantation for chronic liver disease. To determine the effects of mild $\mathrm{CD}$, the routine assessment trans-thoracic echocardiography reports of 246 patients transplanted 01/2007-03/2010 were reviewed. To determine the effects of moderate $\mathrm{CD}$, patients transplanted from 1994-present were highlighted from a prospectively collected database.

Results Mild CD $11.4 \%, 11.4 \%, 2.8 \%$, and $0.8 \%$ of the 246 patients undergoing routine assessment echocardiography had left atrial dilatation, or mild mitral regurgitation, aortic regurgitation or aortic stenosis, respectively. After adjusting for confounding variables there was no association between left atrial dilatation $(p=0.738)$ or mild mitral regurgitation $(p=0.234)$ and a cardiac event post transplant. Similarly, there was no association between left atrial dilatation $(p=0.146)$ or mild mitral regurgitation $(p=0.157)$ and post transplant survival. Moderate CD Ten patients were transplanted with moderate $\mathrm{CD}$ : aortic valve replacement (4), moderate valvular dysfunction (2), HOCM (2), moderate left ventricular failure (1), mitral valvuloplasty (1). Two of these patients underwent simultaneous valve replacement surgery. Of these two patients, one had a peri-operative cardiac event and both were alive at 1-year. For the remaining eight patients who did not undergo intervention the cumulative incidence of a cardiac event by 1-year after transplantation was $68.7 \%$, and the estimated 1 -year survival was $46.7 \%$. All three patients with a pre-existing aortic valve replacement that was not re-done had a cardiac complication: one patient developed bacterial endocarditis and awaits further valve replacement surgery, two died of cardiac failure at 90- and 200-days post transplant.

Conclusion Chronic liver disease patients with co-existent moderate $\mathrm{CD}$ who receive a favourable cardiac risk assessment have greater than expected morbidity and mortality following liver transplantation. Our findings suggest that the severity of non-ischaemic $\mathrm{CD}$ is underestimated in this setting.

Competing interests None declared. 